\title{
Interaction between Uneven Cavity Length and Shaft Vibration at the Inception of Synchronous Rotating Cavitation
}

\author{
Y. Yoshida, ${ }^{1}$ Y. Kazami, ${ }^{2}$ K. Nagaura, ${ }^{3}$ M. Shimagaki, ${ }^{1}$ Y. Iga, $^{2}$ and T. Ikohagi ${ }^{2}$ \\ ${ }^{1}$ Space Transportation Propulsion Research and Development Center, Space Transportation Mission Directorate, \\ Japan Aerospace Exploration Agency, 1 Koganezawa, Kimigaya, Kakuda 981-1525, Japan \\ ${ }^{2}$ Institute of Fluid Science, Tohoku University, 2-1-1 Katahira, Aoba, Sendai, Miyagi 980-8577, Japan \\ ${ }^{3}$ Foundation for Promotion of Japanese Aerospace Technology, 1 Koganezawa, Kimigaya, Kakuda, Miyagi 981-1525, Japan \\ Correspondence should be addressed to Y. Yoshida, yoshida.yoshiki@jaxa.jp
}

Received 23 April 2008; Accepted 7 July 2008

Recommended by Yasutomo Kaneko

\begin{abstract}
Asymmetric cavitation is known as one type of the sources of cavitation induced vibration in turbomachinery. Cavity lengths are unequal on each blade under condition of synchronous rotating cavitation, which causes synchronous shaft vibration. To investigate the relationship of the cavity length, fluid force, and shaft vibration in a cavitating inducer with three blades, we observed the unevenness of cavity length at the inception of synchronous rotating cavitation. The fluid force generated by the unevenness of the cavity length was found to grow exponentially, and the amplitude of shaft vibration was observed to increase exponentially. These experimental results indicate that the synchronous shaft vibration due to synchronous rotating cavitation is like selfexcited vibrations arising from the coupling between cavitation instability and rotordynamics.
\end{abstract}

Copyright ( $\odot 2008$ Y. Yoshida et al. This is an open access article distributed under the Creative Commons Attribution License, which permits unrestricted use, distribution, and reproduction in any medium, provided the original work is properly cited.

\section{INTRODUCTION}

For a turbopump, an inducer is placed upstream of the main impeller to obtain high suction performance, and thus it is one of the key components of a liquid propellant rocket engine. Unfortunately, under some operating conditions, cavitation instabilities such as rotating cavitation or cavitation surge are observed $[1,2]$. In addition to these cavitation instabilities, synchronous rotating cavitation with uneven cavities on each blade is often observed in three-bladed inducers at relatively low cavitation numbers. Synchronous rotating cavitation results in severe synchronous shaft vibration and causes degradation of suction performance due to an asymmetric cavity pattern [2-5].

Previous studies on the relationship between synchronous rotating cavitation and shaft vibration have been conducted by Hashimoto et al. [6] and Kobayashi [5], who found that there is a significant correlation between the amplitude of the shaft vibration and the onset of synchronous rotating cavitation. In addition, Fujii et al. [7] examined such an unsteady phenomenon at lower cavitation numbers through measurements of the pressure fluctuation and blade stress fluctuation in which the pattern of the cavity on each blade changes irregularly while the overall asymmetric pattern is maintained.

The interaction between uneven cavity length and shaft vibration under synchronous rotating cavitation, however, is poorly understood. To clarify the interaction and the origin of this complicated instability, we investigate the growth of the cavity and the shaft vibration in detail with a focus on the inception of synchronous rotating cavitation. We also estimated the fluid force on the inducer based on measurement of unsteady pressure distributions and compared it with the amplitude of the shaft vibration.

\section{EXPERIMENTAL FACILITY AND APPARATUS}

\subsection{Experimental facility}

The experiment was conducted in the Cryogenic Inducer Test Facility (CITF) at Kakuda Space Center of the Japan Aerospace Exploration Agency (JAXA). Figure 1 shows a schematic of the test facility. This facility has two tanks, one upstream (run-tank) and one downstream (catch-tank). The temperature of liquid nitrogen as a working fluid is $74 \mathrm{~K}$ measured at the inducer inlet. 


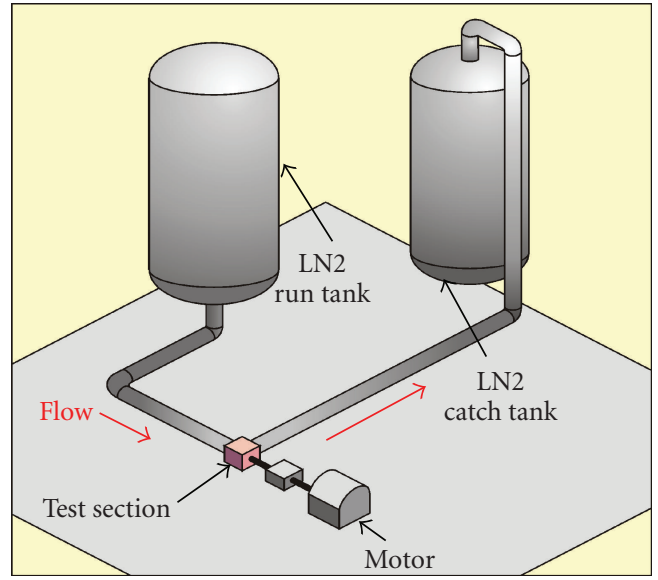

FIGURE 1: Schematic diagram of the cryogenic inducer test facility.

The test inducer has three blades with sweep cutback at the leading edge. Its solidity is about 2.1. The diameter is about $160 \mathrm{~mm}$, and the blade geometry is modified helix. The inducer is driven by an electric motor with an epicyclic gear at a rotation speed of $18300 \mathrm{rpm}$, equal to that of an actual turbopump, and the flow rate is set to remain constant at $Q / Q d=1.06$. The rotor is supported by selflubricated angular contact ball bearings arranged in two spring-preloaded pairs at the inducer side and motor side.

\subsection{Experimental apparatus}

To investigate the behavior of cavitation, it is better to observe the cavitation directly. However, it is difficult to visualize the cavitation at the inducer in cryogenic fluid, that is, nitrogen. Therefore, to estimate the cavitating state of the inducer indirectly, eight pressure sensors (pos. 1-pos. 8) are installed along the blade from the leading edge to the trailing edge on the casing as shown in Figures 2 and 3 [8].

Pressure sensors detect the cavitation near the blade tip, that is, tip leakage vortex cavitation. However, these sensors are unable to detect the cavitation in the backflow upstream of the inducer, that is, backflow vortex cavitation. From previous studies, it is well known that the cavitation near the blade tip considerably affects suction performance and cavitation instability. In addition, the blade cavitation at mid-span seldom appears. Thus, it is reasonable that the cavity length near the blade tip is regarded as an "indication" of cavitation.

Using these pressure sensors at each location, contour plots describing the cavitation state in the blade channel are obtained. Figure 4 was drawn by rearrangement of the wave forms of all the sensors. When a cavitation occurs, the pressure reaches at the saturated vapor pressure as blue region in Figure 4. Therefore, the blue area in these figures shows "estimated cavity region." Using these figures at each cavitation number, the cavity length can be observed by this indirect visualization. We defined the length from the leading edge to trailing edge of the estimated cavity region as "cavity length (Lc)."

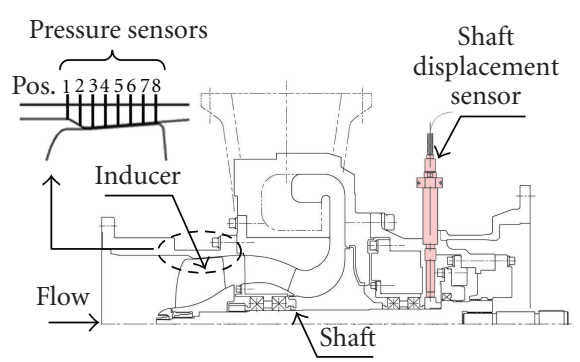

FIgure 2: Schematic diagram of the test section showing the locations of pressure sensors and shaft displacement sensors.

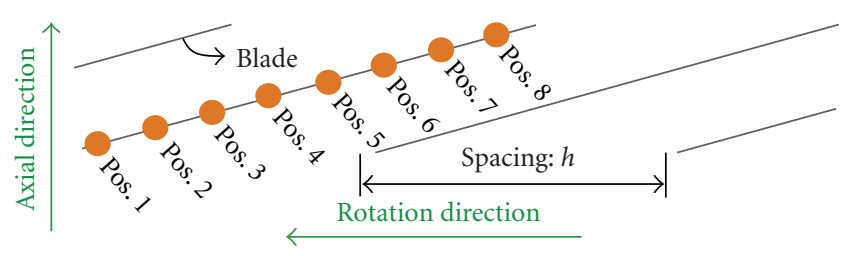

FIGURE 3: Development view of the inducer showing locations of pressure sensors along the inducer blade.

Two shaft displacement sensors for $X-Y$ directions are installed at the backend of the rotor as shown in Figure 2. The sensor for shaft vibration should be installed near the inducer. However, there is no space around the inducer due to the limitation of the pump structure.

\section{ROTATING CAVITATION}

Rotating cavitation is cavitation instability, in which the cavity lengths become uneven on the three blades. There are two types of such cavitation, namely, "super-synchronous" and "synchronous" rotating cavitation. In the first type, as shown in Figure 5(a), the pattern of cavity unevenness propagates from a blade to an adjacent blade, while in the latter type as shown in Figure 5(b), the cavity remains on synchronous rotation. The super-synchronous rotating cavitation is an unsteady phenomenon in which unevenness of the cavity propagates at a speed 1.1-1.2 times higher than that of inducer rotation. On the other hand, synchronous rotating cavitation is a steady asymmetric phenomenon in which unevenness of the cavity does not propagate.

\section{EXPERIMENTAL RISULTS}

\subsection{Head coefficient, cavity length, and shaft vibration}

In a previous work [9], the relation of the cavitation performance, cavity length, and shaft vibration has been examined. The diamond-shaped symbols $(\diamond)$ in Figure 6 show variations of nondimensional cavity length $(\mathrm{Lc} / h)$ normalized by the blade spacing $(h)$ for each channel (i.e., three channels for the three-bladed inducer). The circles (o) in Figure 6 show variations of the inducer head coefficient $\left(\psi / \psi_{0}\right)$ normalized by the reference head coefficient $\left(\psi_{0}\right)$. In addition, the triangles $(\Delta$ and $\Delta)$ in Figure 6 show the 


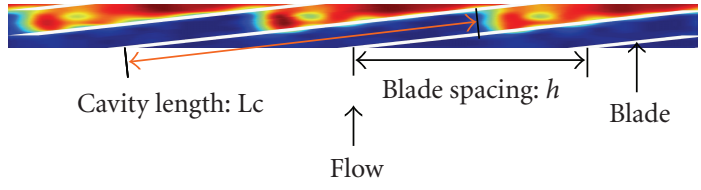

Estimated cavity region

FIgURE 4: Example of estimated cavity region obtained from the measured pressure distribution.

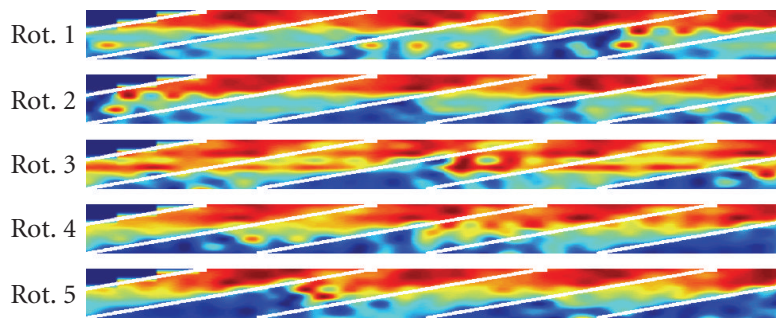

Estimated cavity region

(a)

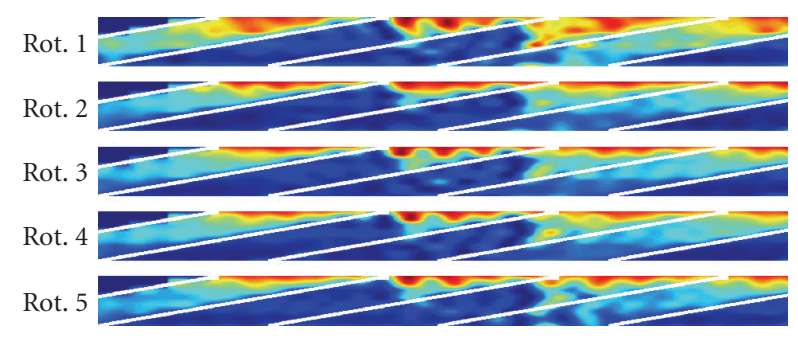

Estimated cavity region

(b)

FIgURE 5: (a) Examples of estimated cavity region under supersynchronous rotating cavitation for every rotation. (b) Examples of estimated cavity region under synchronous rotating cavitation for every rotation.

amplitude of the fluctuating cavity length, and the crosses $(\times)$ show the time averaged cavity length under the supersynchronous rotating cavitation. The average cavity length increases with decreasing of cavitation number. The middle panel of Figure 6 shows shaft vibration detected by the displacement sensors. The crosses $(x)$ in the bottom of Figure 6 show the propagation speed ratio $(\omega / \Omega)$ of rotating cavitation.

As the cavitation number $(\sigma)$ decreases, super-synchronous rotating cavitation occurs at a cavity length of about $\mathrm{Lc} / h=0.5$ (at $\left.\sigma / \sigma_{0}=0.85\right)$. Under such cavitation, cavity length fluctuates on three blades with a certain phase lag, as shown in Figure 7(a). As a result, it can be seen that the unevenness of cavity length propagates in the same direction as the rotor with a speed ratio of $\omega / \Omega=1.1-1.2$, and

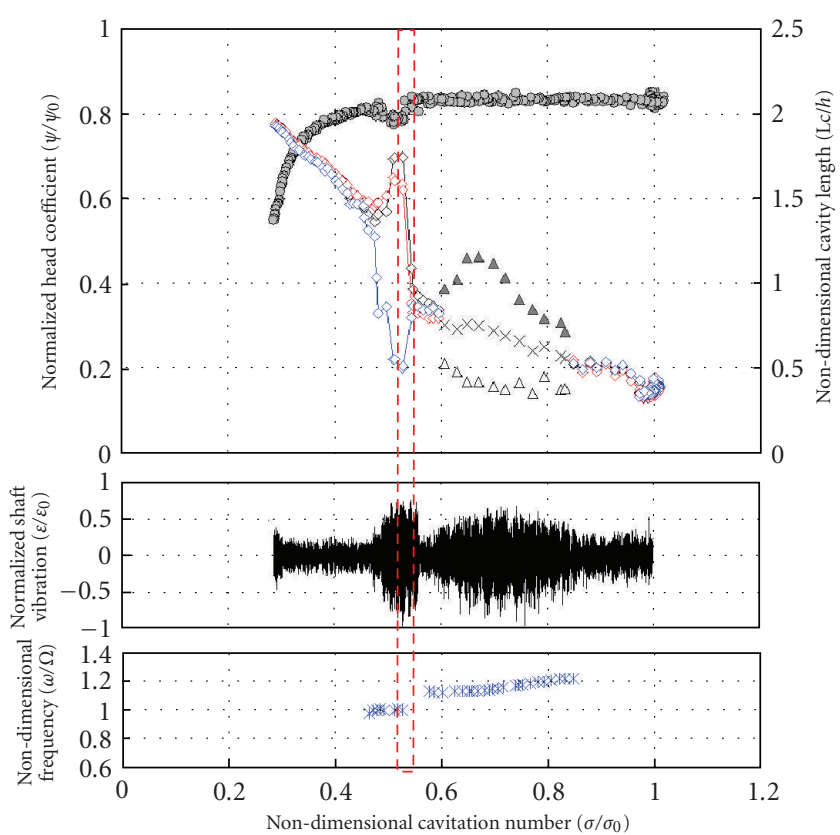

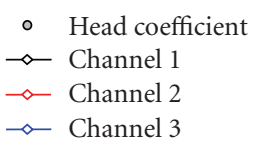

$\times$ Average cavity length

$\Delta$ Maximum cavity length

$\Delta$ Minimum cavity length

FIGURE 6: Cavitation performances, cavity length of each channel (top), shaft vibration (middle), and variations of frequency of shaft vibration (bottom).

that super-synchronous shaft vibration $(\omega / \Omega=1.1-1.2)$ is generated due to the unevenness of the cavity length, as shown in the bottom of Figure 6.

In contrast, synchronous rotating cavitation occurs at a cavity length of $\mathrm{Lc} / h \cong 0.9$ (at $\sigma / \sigma_{0}=0.55$ ) and disappears at a cavity length of $\mathrm{Lc} / h \cong 1.5$. Therefore, the trigger of super-synchronous/synchronous rotating cavitation must be governed only by the cavity length, which is inferred to be one type of fluid dynamic instability with cavitation. This result agrees with the theoretical analysis of the alternate blade cavitation calculated by Horiguchi et al. [10]. They reported that the alternate blade cavitation commences when the cavity length approaches $\mathrm{Lc} / h \cong 0.65$ in an even number of blade cascades. Under synchronous rotating cavitation, the unevenness of cavity length appears clearly as three lengths (short, long, and long) in Figure 7(b). The cavity pattern remains uneven on each blade. Unevenness of cavity length under synchronous rotating cavitation is greater than that under super-synchronous rotating cavitation.

In addition, the circles (o) in Figure 6 show the variations of the inducer head. Within the range of the occurrence of super-synchronous rotating cavitation, the inducer head is not affected by this cavitation instability. However, the inducer head temporarily decreases within the range of the occurrence of synchronous rotating cavitation. After breaking away from the synchronous rotating cavitation, cavity lengths become completely equal near $\mathrm{Lc} / h=1.5$, and 


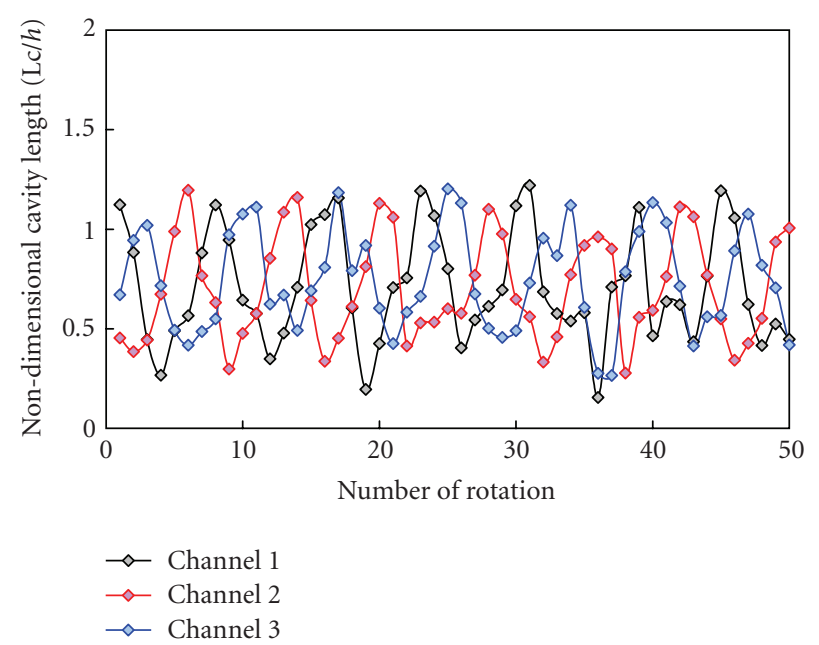

(a)

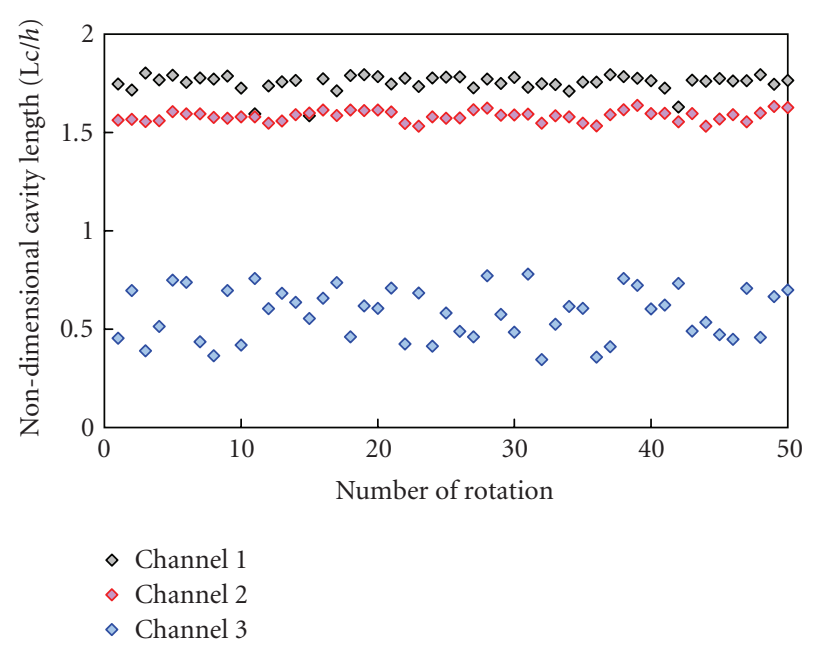

(b)

FIGURE 7: (a) Fluctuations of cavity length under super-synchronous rotating cavitation. (b) Fluctuations of cavity length under synchronous rotating cavitation.

then the head break begins gradually. This means that the inducer head is strongly dependent on the cavity length.

\subsection{Behavior of synchronous rotating cavitation}

As mentioned above, the synchronous rotating cavitation causes inducer head loss and synchronous shaft vibration. We now focus on the growth of the cavity length at the inception of synchronous rotating cavitation in detail to examine its mechanism.

Figure 8 shows the behavior of uneven cavity length at the inception of synchronous rotating cavitation. This figure corresponds to the region bounded by red dashed-line in Figure 6 . The diamond-shaped symbols $(\diamond)$ in Figure 8 show variations of nondimensional cavity length $(\mathrm{Lc} / h)$ of each channel. To estimate the unbalanced fluid force acted on the inducer, the pressure distribution measured by pressure sensors on the casing is integrated. Therefore, this estimated
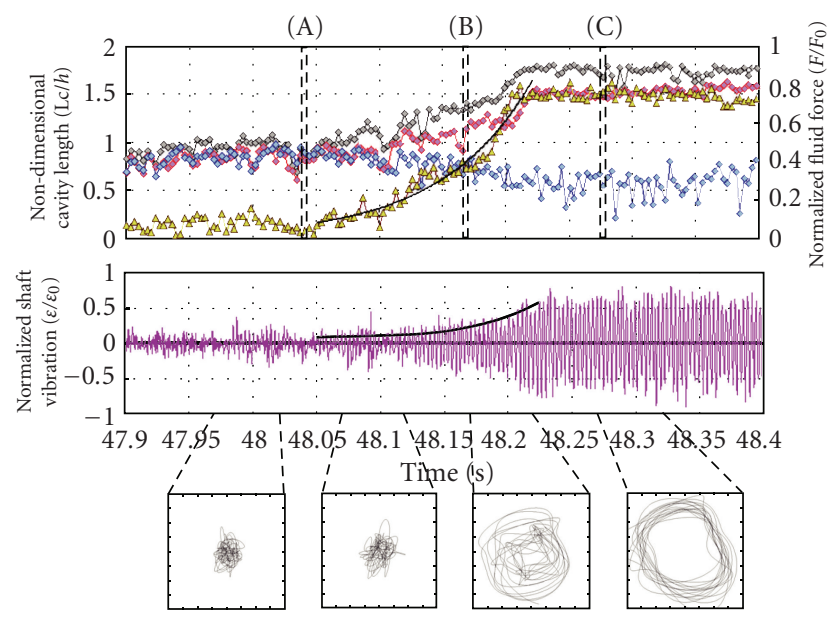

$$
\begin{array}{ll}
\hookleftarrow \text { Channel 1 } & \rightarrow \text { Channel } 2 \\
\neg \text { Channel 3 } & \rightarrow \text { Fluid force }
\end{array}
$$

FIGURE 8: Cavity length of each channel, fluid force (top), the amplitude of shaft vibration (middle), and the Lissajous figures showing the orbit of shaft vibration (bottom).

fluid force is regarded as only an indication of fluid force affecting the inducer. The triangles $(\Delta)$ in Figure 8 show the fluid force $(F)$ normalized by the reference fluid force $\left(F_{0}\right)$. Figure 9 shows estimated cavity region near inception of synchronous rotating cavitation. In Figure 9, the phase of fluid force is indicated by arrows. Under synchronous rotating cavitation, the phase of fluid force is the direction of the channel in which short cavity occurs. The bottom of Figure 8 shows the variation of the shaft vibration during the same time.

Before 48.05 seconds, cavity length, fluid force, and shaft vibration fluctuate slightly, so it should be considered that the synchronous rotating cavitation does not yet occur. After 48.05 seconds, the unevenness of cavity length commences and fluid force also begins to increase, indicating that the onset of synchronous rotating cavitation as cavitation instability is at 48.05 seconds. After 48.22 seconds, the degree of unevenness of cavity length and the unbalanced fluid force is rapidly stop increasing, indicating that the synchronous rotating cavitation develops fully at 48.22 seconds. The rapid growth time (from 48.05 seconds to 48.22 seconds) of the cavity unevenness and the shaft vibration is 0.17 seconds, that is, about fifty rotations.

Under the steady condition of the synchronous rotating cavitation (after 48.22 seconds), cavity lengths of channels 1 and 2 are stable and longer $(\mathrm{Lc} / h>1.5)$, but that of channel 3 is unstable and shorter caused by interaction with the longer cavity in channels 1 and $2(\mathrm{Lc} / h<0.8)$.

In the bottom of Figure 8, the Lissajous figures show the orbit of the shaft vibration from the measurement of the $X-Y$ displacement sensors. The amplitude is small, and the orbit is random prior to the synchronous rotating cavitation (before 48.05 seconds), while after 48.22 seconds, the orbit of the shaft vibration can be visualized as a large limit circle 


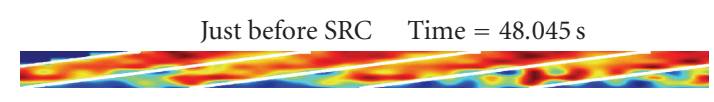

(a)

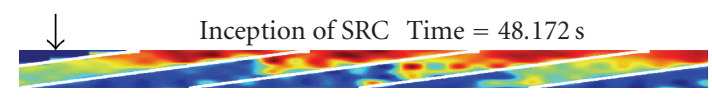

(b)

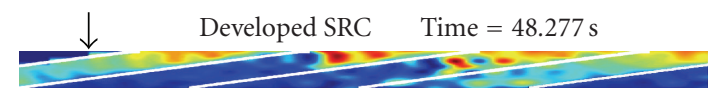

Estimated cavity region

(c)

FIGURE 9: Figures of estimated cavity region near inception of synchronous rotating cavitation (SRC).

under the full developed synchronous rotating cavitation. It seems that the shaft vibration caused by synchronous rotating cavitation is like self-excited vibrations.

\section{DISCUSSION}

\subsection{Growth of synchronous rotating cavitation}

During the growth of the synchronous rotating cavitation in Figure 8, it is observed that the fluid force caused by the unevenness of the cavity length develops exponentially, and that the amplitude of shaft vibration also increases exponentially. The exponential fit curves along the fluid force and the amplitude of shaft vibration are shown in Figure 8 within 48.05-48.22 seconds. To compare the growth behavior of the fluid force with that of the shaft vibration, logarithmic decrements $(\delta)$ are estimated by (1) using these fit curves:

$$
\begin{aligned}
\varepsilon & =\varepsilon^{*} \times e^{-\delta_{\varepsilon}\left(t-t_{\text {inc }}\right) / T} \times \cos \left(\Omega\left(t-t_{\text {inc }}\right)\right), \\
F & =F^{*} \times e^{-\delta_{F}\left(t-t_{\text {inc }}\right) / T}
\end{aligned}
$$

The logarithmic decrement of the shaft vibration, $\delta_{\varepsilon}$, is -0.03 , and that of the fluid force, $\delta_{F}$, is -0.046 . Both logarithm decrements are negative with almost equal values.

Figure 10 shows the amplitude of shaft vibration as a function of the fluid force during the inception of the synchronous rotating cavitation. This figure presents that the amplitude of shaft vibration increases linearly with fluid force. When the amplitude of synchronous shaft vibration increases, the clearance gap between the tips of the rotating inducer and the casing wall varies at each blade. The degree of the tip leakage cavitation strongly depends on the tip clearance [1]. Hence, it is considered that this synchronous shaft vibration is acted upon by the synchronous fluid force through the nonuniform tip leakage cavitation on each blade, that is, synchronous rotating cavitation.

From these results, this synchronous shaft vibration is indicated to be like self-excited vibrations with a coupling between the synchronous rotating cavitation and the rotor

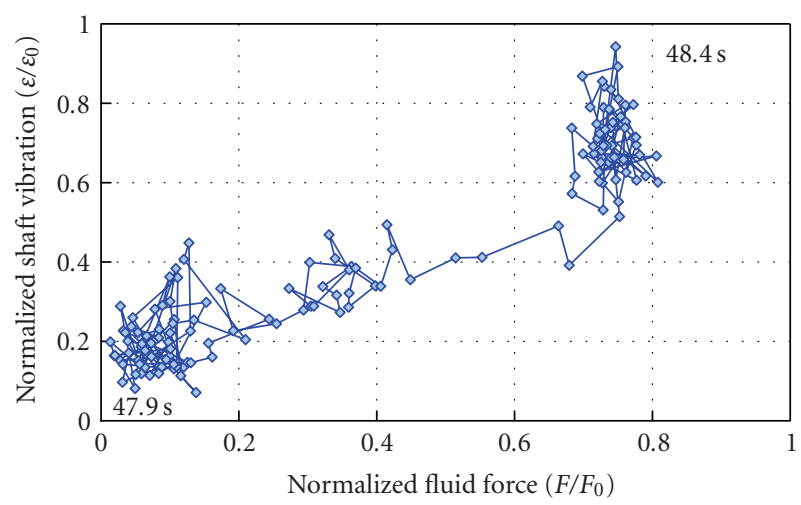

FIGURE 10: Increase of the amplitude of shaft vibration as a function of the fluid force.

vibration, although rotordynamic fluid forces caused by cavitation have long been known to be the self-sustaining force of rotordynamics [3].

\subsection{Hydrodynamics-rotordynamics coupling mechanism}

From the relation of cavity length, fluid force and shaft vibration mentioned above, the estimated mechanism of the development of synchronous rotating cavitation is presented in Figure 11. First, when the cavity length reaches a critical length, cavitation instability occurs as flow field dynamics [10]. The equal cavity length of the three blades becomes uneven, that is, this is the start of the synchronous rotating cavitation. Nonuniform pressure and velocity distribution around the inducer caused by the uneven cavity length generate the fluid force acting on the inducer. Second, this fluid force results in rotor displacement and whirl motion in rotordynamics. This whirl motion creates nonuniform tip clearance, which results in the acceleration of cavity unevenness associated with leakage flow in the nonuniform tip clearance. As this process is repeated, synchronous rotating cavitation grows. This hydrodynamics (with cavitation)rotordynamics coupling mechanism in the inducer is similar to the aerodynamic-rotordynamic coupling mechanism in an axial compressor [11] with regard to the effect of nonuniform tip clearance. This mechanism is shown as a picture in Figure 12.

On the other hand, Bhattacharyya et al. [12] experimentally investigated the rotordynamic forces in a whirling cavitating inducer, and d'Agostino et al. [13] also analyzed it. Their results showed the occurrence of large destabilizing peaks in the tangential force acting on the whirl orbit for a positive frequency ratio $(\omega / \Omega \cong 0.2)$ at a certain cavitation condition. However, there have been no reported results concerning the whirl frequency ratio at $\omega / \Omega=1.0$, that is, synchronous rotating cavitation. Therefore, to more fully clarify the mechanism of synchronous rotating cavitation, an extension of those experiments is underway using Ebara Rotordynamic Test Stand (EBARTS) [14], which will add to our understanding of the hydrodynamics (with cavitation)rotordynamics coupling mechanism. 


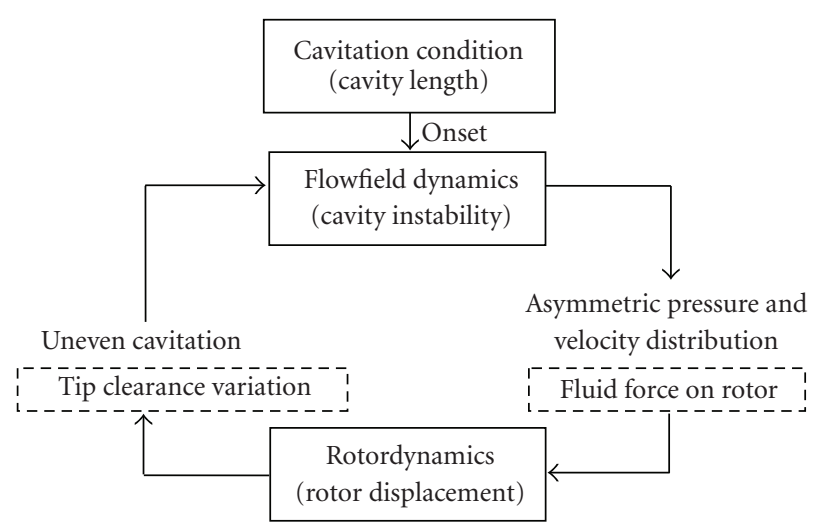

Figure 11: Hydrodynamics (cavitation)—rotor-dynamics coupling mechanism.

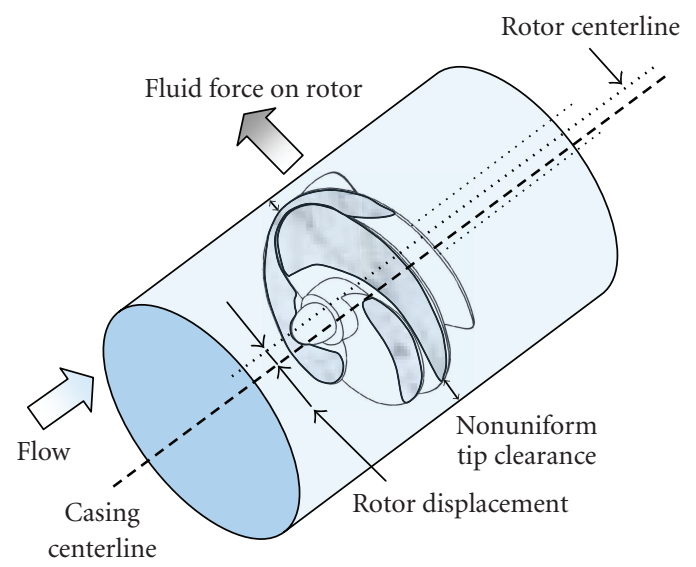

FIGURE 12: Figure of hydrodynamics (cavitation)—rotordynamics coupling mechanism.

\section{CONCLUSIONS}

By experimental investigation of the relationship of the cavity length, fluid force, and shaft vibration at the inception of synchronous rotating cavitation, the following points were clarified.

(1) The growth time of the cavity unevenness and the shaft vibration at the inception of synchronous rotating cavitation is only fifty rotations.

(2) Fluid force caused by the unevenness of the cavity length develops exponentially, as does the amplitude of shaft vibration.

(3) The logarithm decrement of the amplitude of shaft vibration is almost equal to that of the fluid force.

(4) The synchronous shaft vibration due to the synchronous rotating cavitation seems to be like selfexcited vibrations, which is coupled hydrodynamics (cavitation instability) with rotordynamics in Figure 11.

\section{NOMENCLATURE}

$F$ : Fluid force

$h$ : Spacing

Lc: Cavity length

Q: Flow rate

$Q d$ : Design flow rate

$T$ : Cycle of one rotation

$\delta$ : Logarithmic decrement

$\varepsilon: \quad$ Amplitude of shaft vibration

$\varepsilon_{0}:$ Reference amplitude of shaft vibration

$\sigma: \quad$ Cavitation number

$\sigma_{0}$ : Reference value of cavitation number

$\Omega:$ Angular velocity of rotor

$\omega$ : Angular velocity of rotating cavitation

$\psi$ : Head coefficient

$\psi_{0}$ : Reference value of head coefficient.

\section{ACKOWLEDGMENTS}

The authors would like to thank Mr. Yoshifumi Sasao of Tohoku University, Mr. Hirotaka Kure of IHI Co., Ltd., Tokyo, Japan, and Masato EGUCHI of Ebara Research Co., Ltd., Kanagawa, Japan, for useful discussions and their great help with data processing.

\section{REFERENCES}

[1] A. J. Acosta, "An experimental study of cavitating inducers," in Proceedings of the 2nd Symposium on Naval Hydrodynamics (ONR/ACR '58), pp. 537-557, Washington, DC, USA, August 1958.

[2] K. Kamijo, T. Shimura, and M. Watanabe, "An experimental investigation of cavitating inducer instability," ASME Paper 77-WA/FW-14, 1977.

[3] W. Rosenmann, "Experimental investigations of hydrodynamically induced shaft forces with a three-bladed inducer," in Proceedings of ASME Symposium on Cavitation in Fluid Machinery, pp. 172-195, Chicago, Ill, USA, November 1965.

[4] Y. Tsujimoto, Y. Yoshida, Y. Maekawa, S. Watanabe, and T. Hashimoto, "Observations of oscillating cavitation of an inducer," Journal of Fluids Engineering, vol. 119, no. 4, pp. 775781, 1997.

[5] S. Kobayashi, "Effects of shaft vibration on occurrence of asymmetric cavitation in inducer," JSME International Journal. Series B, vol. 49, no. 4, pp. 1220-1225, 2006.

[6] T. Hashimoto, M. Yoshida, M. Watanabe, K. Kamijo, and Y. Tsujimoto, "Experimental study on rotating cavitation of rocket propellant pump inducers," AIAA Journal of Propulsion and Power, vol. 13, no. 4, pp. 488-494, 1997.

[7] A. Fujii, S. Azuma, M. Uchiumi, Y. Yoshida, and Y. Tujimoto, "Unsteady behavior of asymmetric cavitation in a 3-bladed inducer," in Proceedings of the 5th International Symposium on Cavitation (CAV'03), Osaka, Japan, November 2003.

[8] Y. Yoshida, K. Kikuta, S. Hasegawa, M. Shimagaki, and T. Tokumasu, "Thermodynamic effect on a cavitating inducer in liquid nitrogen," Journal of Fluids Engineering, vol. 129, no. 3, pp. 273-278, 2007.

[9] Y. Yoshida, Y. Sasao, M. Watanabe, T. Hashimoto, Y. Iga, and T. Ikohagi, "Thermodynamic effect on rotating cavitation in an inducer," in Proceedings of ASME Fluid Engineering Division 
Summer Meeting (FEDSM '07), San Diego, Calif, USA, JulyAugust 2007.

[10] H. Horiguchi, S. Watanabe, Y. Tsujimoto, and M. Aoki, "A theoretical analysis of alternate blade cavitation in inducers," Journal of Fluids Engineering, vol. 122, no. 1, pp. 156-163, 2000.

[11] A. A. Al-Nahwi, J. D. Paduano, and S. A. Nayfeh, "Aerodynamic-rotordynamic interaction in axial compression systems-part II: impact of interaction on overall system stability," Journal of Turbomachinery, vol. 125, no. 3, pp. 416424, 2003.

[12] A. Bhattacharyya, A. J. Acosta, C. E. Brennen, and T. K. Caughey, "Rotordynamic forces in cavitating inducers," Journal of Fluids Engineering, vol. 119, no. 4, pp. 768-774, 1997.

[13] L. d'Agostino, F. d'Auria, and C. E. Brennen, "A threedimensional analysis of rotordynamic forces on whirling and cavitating helical inducers," Journal of Fluids Engineering, vol. 120, no. 4, pp. 698-704, 1998.

[14] M. Eguchi and Y. Maruta, "Development of rotordynamics measurement system with active magnetic bearings," in Proceedings of the 10th Asia-Pacific Vibration Conference (APVC '03), Gold Coast, Australia, November 2003. 

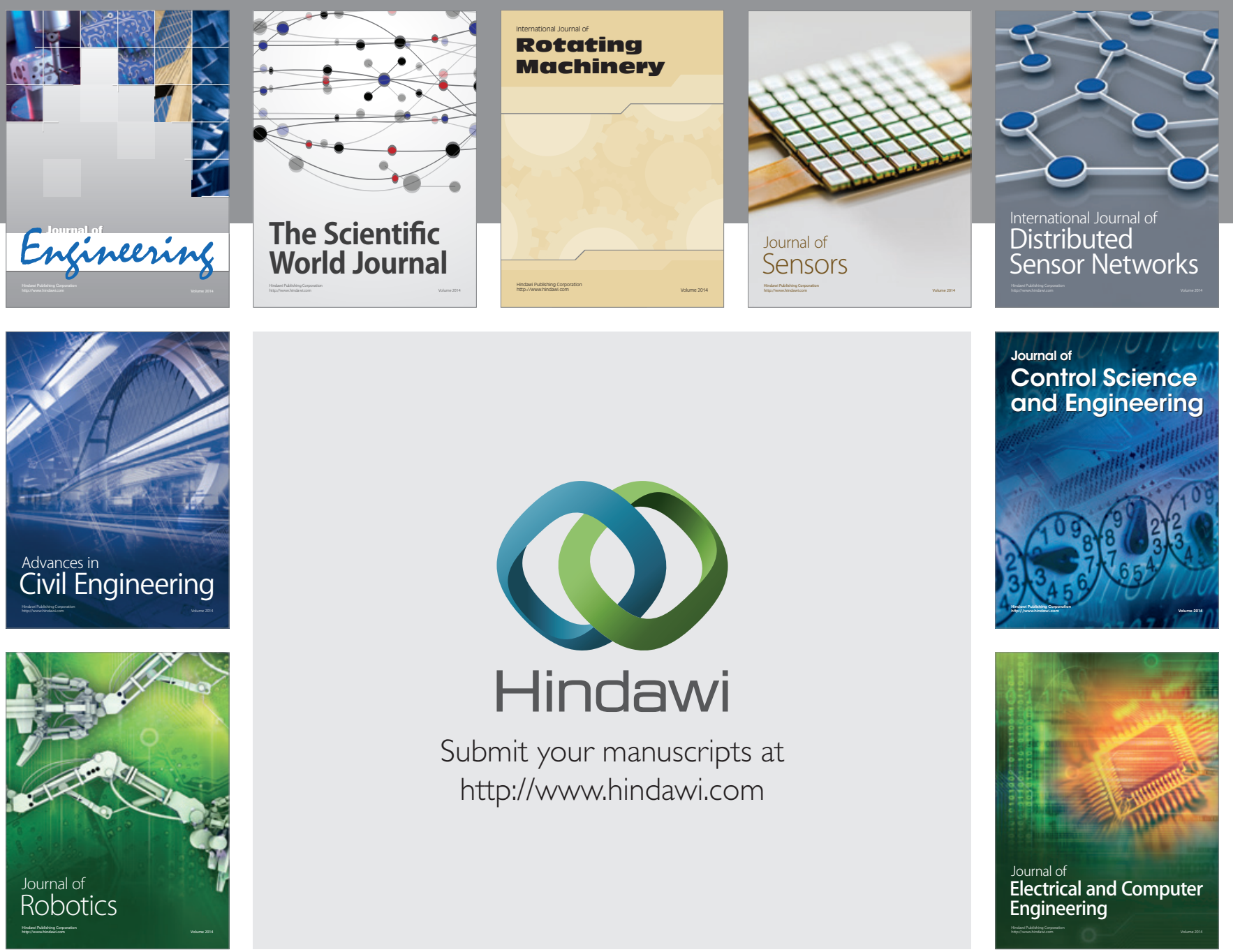

Submit your manuscripts at

http://www.hindawi.com
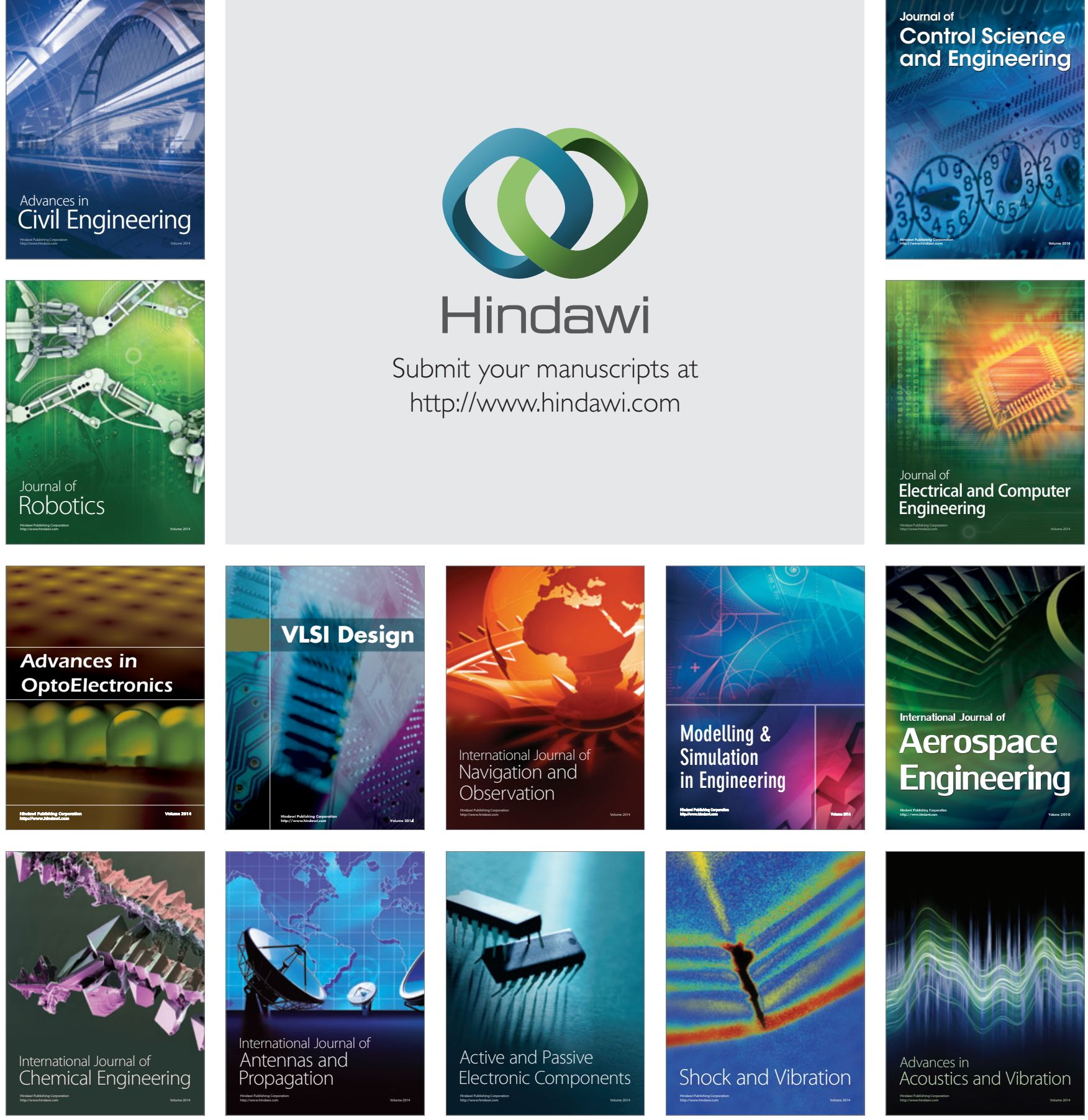